\title{
Conductance-Based Refractory Density Approach for a Population of Bursting Neurons
}

\author{
Anton Chizhov ${ }^{1,2,3}$, Fabien Campillo ${ }^{3,4}$, \\ Mathieu Desroches ${ }^{3,4}$, Antoni \\ Guillamon $^{3,5}$, Serafim Rodrigues ${ }^{3,6}$
}

the date of receipt and acceptance should be inserted later

Acknowledgements This research is supported by the Basque Government through the BERC 2018-2021 program and by the Spanish State Research Agency through BCAM Severo Ochoa excellence accreditation SEV-2017-0718 and through project RTI2018-093860-B- C21 funded by (AEI/FEDER, UE) and acronym "MathNEURO". SR would further like to acknowledge the support of Ikerbasque (The Basque Foundation for Science). Moreover, the research of AC was supported by the Russian Science Foundation grant (project 16-1510201). The research of AG was supported by the Spanish grant MINECO-FEDER-UE MTM-2015-71509-C2-2-R and the Catalan Grant number 2017SGR1049.

\footnotetext{
Abstract The conductance-based refractory density (CBRD) approach is a parsimonious mathematical-computational framework for modeling interacting populations of regular spiking neurons, which, however, has not been yet extended for a population of bursting neurons. The canonical CBRD method allows to describe the firing activity of a statistical ensemble of uncoupled Hodgkin-Huxley-like neurons (differentiated by noise) and has demonstrated its validity against experimental data. The present manuscript generalises the CBRD for a population of bursting neurons; however, in this pilot computational study we consider the simplest setting in which each individual neuron

Anton Chizhov

1) Ioffe Institute,

E-mail: anton.chizhov@mail.ioffe.ru

Politekhnicheskaya str., 26, 194021, St.-Petersburg, Russia

2) Sechenov Institute of Evolutionary Physiology and Biochemistry of Russian Academy of Sciences, Torez pr., 44, 194223, St.-Petersburg, Russia

Tel.: +7-812-2927145

Fax: +7-812-2971017

3) BCAM - Basque Center for Applied Mathematics, Bilbao, Bizkaia, Spain

Fabien Campillo \& Mathieu Desroches

4) Inria Sophia Antipolis Méditerranée, MathNeuro Team, Sophia Antipolis, France

Antoni Guillamon

5) Universitat Politècnica de Catalunya, Departament de Matemàtiques, Barcelona, Spain

Serafim Rodrigues

6) Ikerbasque, Basque Foundation for Science

E-mail: srodrigues@bcamath.org
} 
is governed by a piecewise linear bursting dynamics. The resulting population model makes use of slow-fast analysis, which leads to a novel methodology that combines CBRD with the theory of multiple timescale dynamics. The main prospect is that it opens novel avenues for mathematical explorations, as well as, the derivation of more sophisticated population activity from Hodgkin-Huxley-like bursting neurons, which will allow to capture the activity of synchronised bursting activity in hyper-excitable brain states (e.g. onset of epilepsy).

Keywords neuronal population; conductance-based refractory density model; intrinsically bursting neuron

\section{Introduction}

An intense area of research in mathematical and computational neuroscience is the study of population dynamics of excitable neurons within a neuronal tissue as observed via electro-chemical observables. Advancing this topic constitutes a critical step which, if further progress is made, will provide insights as to how the brain orchestrates information and performs computations. Assuming the postulates put forth by the Hodgkin-Huxley formalism then it is arguably safe to say that single neuron activity is computationally understood. From a mathematical viewpoint, slow-fast theory has made considerable progress in characterising and classifying precisely the electrical patterns of single neurons. In contrast, the understanding of neuronal population dynamics lags behind and a number of approaches have been proposed to express macroscopic spatio-temporal observables (e.g. LFP, EEG, etc). To name a few, these approaches include Neural Mass models (Freeman (1972, 1975); Breakspear et al. (2005); Rodrigues et al. (2009); Marten et al. (2009)), Firing rate models (Wilson and Cowan (1972); Chizhov et al. (2007)), Neural Fields (Amari (1977); beim Graben and Rodrigues (2013); Avitabile et al. (2016)), the Ott-Antonsen ansatz (Ott and Antonsen (2008); Montbrió et al. (2015)), Population density models (Knight at al. (1996); Brunel and Hakim (1999); Knight et al. (2000); Nykamp and Tranchina (2000); Apfaltrer et al. (2006); Ly and Tranchina (2007, 2009); Chizhov and Graham (2007, 2008)) and Kinetic theory (Ventriglia (1974)). The difficulty in progress is largely due to the fact that neurons are excitable open systems and there is strong coupling between the different temporal and spatial neuronal scales. The complex network synaptic connectivity shows lognormal distribution (with heavy tails) as well as extracellular characteristics. The temporal dynamics have a variety of oscillations (due to the neurons' intrinsic physiological properties).

In general, population density models for complex multidimensional neurons are expressed via multidimensional equations in partial derivatives (PDEs). In large scale simulations, these multidimensional equations can lead to computational bottlenecks. Standard population density models do not attempt to reduce the dimensionality of these models. Presently, one of the stand-out 
paradigms for modelling neuronal populations is the refractory density (RD) approach, which belongs to the class of probability density methods (Knight et al. (2000)). It is as an efficient computational method to simulate neuronal populations (seen as statistical ensembles) of uncoupled neurons receiving similar input and dispersed by noise (or each neuron receiving individual Gaussian noise and a common for all neurons of a population, time-varying input). In first approximation, the RD method is akin to the methods developed in statistical physics, in particular the Boltzmann molecular chaos hypothesis, which allows to uncorrelate elastic collisions between pairs of particles within a gas (due to conservation of momentum). As a consequence, it provides a plausible construct of a macroscopic description of the population by a single density. The RD method was first developed for simple, one-parametric integrate-and-fire like neurons (Eggert and van Hemmen (2001); Gerstner and Kistler (2002)). A significant step forward was taken by showing that the method could be extended for Hodgkin-Huxley like neurons in Chizhov et al. (2006) and Chizhov and Graham (2007), which was then denoted as the conductance-based refractory density (CBRD) approach. The key point for considering Hodgkin-Huxley-like models is because these models have shown to provide good accurate approximations of neuronal firing and bursting as observed in a variety of experimental protocols that not only consider synaptic voltage-independent currents as an input but also synaptic conductances (Fernandez and White (2010); Smirnova et al. (2015)), which is in contrast to the majority of simplified models that generally assume 1D input. These two inputs are crucial, which allow to relate to numerous experimental electrophysiological paradigms, experimental constraints (e.g. on neurophysiological parameters such as conductances) as those imposed in Dynamic-Clamp and therefore addressing questions imposed by these experimental setups. Having said that, we note that progress is being made with new generation of simplified models with adaptive threshold dynamics, which have been shown to reproduce double-electrode current-clamp recordings (Gerstner and Naud (2009)). While, these new generation models can be considered within the framework of the RD approach (Schwalger et al. (2017)), we will still rely on the Hodgkin-Huxley neuron framework. Therefore, an important advantage of the CBRD approach is its computational efficiency, because it reduces the description of Hodgkin-Huxley neuron population to 1D transport equations, whereas the conventional probability density approach formally leads to computationally inefficient multi-dimension PDEs.

The reduction to 1D PDEs in the CBRD approach (see Section 2.2) is possible due to a key insight, which is to parameterise the neuronal state variables by a single phase variable, the time elapsed after spike $t^{*}$ (Chizhov and Graham (2007)). Specifically, the neuronal membrane voltages and possibly other neuronal state variables are functions of only time $t$ and $t^{*}$. This is certainly valid for neurons with a renewal process at a spike (action potential), such as neurons modelled by integrate-and-fire models, spike-response (Gerstner and Kistler (2002)) or fast-spiking neurons (Chizhov et al. (2006)). It is approximately true for neurons with slow gating variables, like adaptive neurons 
(Chizhov and Graham (2007)). This approximation is valid if the relaxation of each adaptive variable between spikes is roughly the same for different neurons within the population, which induces the inter-spike intervals of all neurons to become more or less comparable. Moreover, similarly to the typical RD approaches, an important assumption is to assume that all neurons within a population are governed by the same dynamics and are de-coupled, however are driven by the same input (possibly differentiated by noise). Thus, when a time-dependent input drives (in a similar fashion all neurons within the population), the state variables of the neurons, parameterized by $t^{*}$, fluctuate close to the average value of the population activity. This is the basis to split the problem into the solution of the equations for the average state variables and the fluctuations due to noise. The noise effect is captured by the so-called hazard-function, which evaluates a probability for a single neuron to fire provided that the expected values of its states are known. The approximation of the hazard-function was derived Chizhov and Graham (2007, 2008) by considering certain assumptions on the noise term. Interestingly, it is independent on the neuron model and substitutes former phenomenological approximations.

The CBRD has shown to be capable of modelling coupled/heterogeneous networks where each population node is a different CBRD model and more importantly has shown its validity in experimental studies. For example, a comparison of the CBRD model to a standard mean field EEG model was made, which demonstrated improvement gains (Chizhov et al. (2007)) and subsequently, a number of modelling extensions followed. These included the case of temporally correlated noise (Chizhov and Graham (2008)), two-compartment neurons (Chizhov (2014)), lognormal distribution of input weights (Chizhov (2017)) and finite-size population (Schwalger et al. (2017); Dumont et al. (2017)). The validity of the CBRD method was also tested against data. In particular, it has modelled the visual cortex activity via two 2D-layers of two-type populations that respond to both electrical and visual stimulation (Chizhov (2014)). It has also provided insights in modelling epileptic discharges, such as, interictal discharges modelled via two populations (Chizhov et al. (2017)), three populations with ionic dynamics for ictal discharges (Chizhov et al. (2019b)), 2D-lattice of two-type populations for spreading interictal discharges (Chizhov et al. (2019)).

In the present paper we want to show that CBRD can be further extended to capture more complex electrical oscillations like bursting. Bursting neurons are characterised by the alternation between two distinct activity regimes, namely quiescent phases where the voltage slowly follows a quasi-steady-state and burst phases where groups of spikes are fired consecutively. There are two main modelling frameworks to capture bursting dynamics. The first framework corresponds to smooth continuous-time so-called slow-fast or singularly perturbed dynamical systems with at least three state variables, two fast and one slow. Indeed, in the context of geometric singular perturbation theory (GSPT) (Fenichel (1979); Jones (1995)), the so-called fast subsystem, obtained as the slow variable is frozen and considered as a parameter, is a planar dynamical system with one distinguished parameter (the frozen slow variable). This pla- 
nar system displays bistability between stable limit cycles and stable equilibria for a range of values of the frozen slow variable. What is more, this bistable region of the bifurcation diagram of the fast subsystem possesses a hysteretic loop, that is, a pair of bifurcations which connect in parameter space both families of attractors, equilibria and limit cycles. Hence, in the full system, the dynamics of the slow variable is organised (often through a feedback term involving one of the two fast variables) so that it oscillates in the region of bistability and switches between quasi-stationary (quiescent phase) to quasiperiodic (burst phase) regimes when slowly passing near the bifurcations of the fast subsystem that organises this hysteretic loop. Therefore, the two fast variables of a smooth bursting model account for the burst phase and the slow variable for the quiescent phase while also driving slowly (on average) the membrane potential through the burst; for more details see Rinzel (1986); Izhikevich (2000); Desroches and Rodrigues (2018).

The second framework is that of hybrid dynamical systems, which are effectively a combination of smooth continuous-time dynamical systems together with a map. This map is applied to one or several variables each time they reach a predefined value (threshold), at which point the continuous-time system stops and these variables are mapped (instantaneously) to new values or resets. Classical example of hybrid neuron models are integrate-and-fire model (Knight (1972)) where typically only the membrane potential gets reset upon threshold, which provides a spiking model with only one state variable (voltage). In the case of bursting neurons, hybrid models have two variables which both undergo reset upon threshold: membrane potential and a recovery variable which mimics gating dynamics. In this work, we consider for simplicity the hybrid dynamical systems framework, but with an outlook for future work with the smooth case.

It is worth remarking that previous studies using the RD approach have also considered bursting neurons. However, we note that in these studies (for example, Casti et al. (2002)) the derivation of the RD model from a simple bursting neuron retains the dimensionality of the neuronal equations. Moreover, an extension of this approach to more complex neurons would increase the dimension even more, which implies a loss in computational efficiency. In contrast, the CBRD approach always reduces the dimensionality, while fully representing the fast and slow variables of the underlying neuron model and therefore allows to model intrinsic bursting and is applicable to coupled populations and therefore offers significant advantages. To compare, in the setting of bursting dynamics with hybrid models the $\mathrm{RD}$ approach gives rise to a 2D transport equation (as opposed to 1D transport for spiking regime). Moreover, the two state variables are parameterised by the time since last spike and the time since last burst. Specifically, the transport equations correspond to these two main state variables while the neuronal density describes neuronal dynamics and dispersion across population due to noise. The loss of computational efficiency in this case is probably the main reason as to why such $2 \mathrm{D}$ approach has not been developed (or attempted). However, we note that the dissipation is negligible during bursts because of their relatively short duration 
and high conductance state that shunts the noisy currents. We make use of this property (as an advantage) to reduce the system to one-dimensional and as a consequence we obtain a computationally effective 1D RD model for a population of bursting neurons. The present work has to be seen as a pilot computational study, where for simplicity we model bursting dynamics with hybrid models. However, we envisage that this work will stimulate the use of smooth slow-fast systems and will provide future challenging mathematical questions as well as the possibility of testing experimental data from bursting populations.

\section{Methods}

\subsection{Single neuron model: leaky integrate-and-fire neuron with noise}

The framework setting under which we start our work is with hybrid dynamical systems but with the future outlook to develop and enhance it to smooth continuous-time slow-fast systems and their corresponding conductance (Hodgkin-Huxley) equivalent models. To this end, our starting point is the LIF spiking neuron model (i.e. hybrid dynamical system) since we will show that when we pass onto the bursting hybrid model, many of the LIF features will be retained within the macroscopic refractory density formulation. Specifically, the LIF neuron is given by the equation

$$
C \frac{d V}{d t}=-\left(g_{L}+s(t)\right)\left(V-V_{\text {rest }}\right)+I(t)+\sigma_{I} \xi(t),
$$

where $\xi(t)$ is a Gaussian white noise process characterised by its mean value, $\langle\xi(t)\rangle=0$, and auto-correlation $\left\langle\xi(t) \xi\left(t^{\prime}\right)\right\rangle=C / g_{L} \delta\left(t-t^{\prime}\right)$; the standard deviation, $\sigma_{I}$, is the noise amplitude. The neuron fires when the potential $V$ crosses a threshold $U_{T}$. Immediately after a spike, $V$ is reset to $V_{\text {reset }}$. The LIF neuron is characterised by the capacitance $C$ and the leak conductance $g_{L}$. The input is determined by two signals, the synaptic current measured at the voltage level equal to $V_{\text {rest }}, I(t)$, and the total synaptic conductance $s(t)$. The effective membrane time constant is $\tau_{m}=C /\left(g_{L}+s(t)\right)$.

We can then derive the equations of motion for a population of an infinite number of eq.(1)-based LIF neurons receiving a common 2D input $[I(t), s(t)]$ and noise, individual for each neuron. The population firing rate is defined as a sum of all spikes, $n^{a c t}$, from neurons of the population over a short time window $\Delta t$, divided by the number of neurons $N$. After taking the limits of $N \rightarrow \infty$ and $\Delta t \rightarrow 0$, the firing rate $\nu$ is obtained as

$$
\nu(t)=\lim _{\Delta t \rightarrow 0} \lim _{N \rightarrow \infty} \frac{1}{\Delta t} \frac{n^{a c t}(t ; t+\Delta t)}{N} .
$$

The direct approach to calculate the firing rate is via Monte Carlo simulations. 


\subsection{Population model: CBRD approach for integrate-and-fire neurons}

The firing rate for a population of LIF neurons receiving a common deterministic input and Gaussian noise, is well approximated by the CBRD model Chizhov and Graham (2007), Chizhov and Graham (2008). The CBRD model is expressed by a system of transport equations for two variables: the neuronal density in $t^{*}$-space $\rho\left(t, t^{*}\right)$, so-called refractory density, and $U\left(t, t^{*}\right)$, the membrane potential averaged across noise realizations, where $t^{*}$ is the time elapsed since the last spike. The state of each neuron is parameterized by this phase variable. For the particular case of a LIF-neuron, its only state variable is $V\left(t, t^{*}\right)$ (with its mathematical expectation $U\left(t, t^{*}\right)$ ). (For more complex neuron models the gating variables of ionic channels are also parameterized by $t^{*}$, which allows to preserve the number of independent variables and thus leads to 1D PDEs (see Section 3.4).) This parameterization leads to a reduced description with only one phase variable, which nevertheless preserves the information about neuronal states, provided all neurons they are subject to the same input history. The equation for $U\left(t, t^{*}\right)$ is derived from the original ordinary differential eq.(1) by substituting the total derivative in time by a sum of partial derivatives: $d / d t=\partial / \partial t+\partial / \partial t^{*}$. The equation for $\rho\left(t, t^{*}\right)$ is derived from the neuron number conservation law $\partial \rho / \partial t+d i v \rho v=-\rho H$, where div is the divergence operator in the coordinate space ( $t^{*}$-space); $v$ is the velocity of neuronal flux in the $t^{*}$-space, which is $v \equiv d t^{*} / d t$ and equal to 1 by the definition of $t^{*}$, i.e. $v \equiv 1$. The expression $-\rho H$ is the source term that describes firing, where $H=H\left(U\left(t, t^{*}\right), s(t)\right)$ is the probability for a single neuron to fire and $s(t)$ is a synaptic conductance which together with $I(t)$ drives equally all neurons within a population; for details see our earlier work (Chizhov and Graham (2007)) and further information that is provided below. Finally, the equations for $\rho\left(t, t^{*}\right)$ and $U\left(t, t^{*}\right)$ are as follows:

$$
\begin{aligned}
\frac{\partial \rho}{\partial t}+\frac{\partial \rho}{\partial t^{*}} & =-\rho H\left(U\left(t, t^{*}\right), s(t)\right) \\
C\left(\frac{\partial U}{\partial t}+\frac{\partial U}{\partial t^{*}}\right) & =-\left(g_{L}+s(t)\right)\left(U-V_{\text {rest }}\right)+I(t),
\end{aligned}
$$

where $I$ is the synaptic current; $g_{L}$ is the leak conductance, $C$ is the membrane capacitance and $t^{*}$ is a time parametrization, which denotes the time since last spike. The boundary conditions are

$$
\nu(t) \equiv \rho(t, 0)=\int_{+0}^{\infty} \rho H d t^{*} \quad \text { and } \quad U(t, 0)=V_{\text {reset }},
$$

where $\nu(t)$ is the population firing rate. When calculating the dynamics of a neural population, the integration of eq.(4) defines at each time moment $t$ the distribution of deterministic (i.e. devoid from noise) voltage $U$ along $t^{*}$. Then, the effect of threshold crossing and diffusion by noise are taken into account by 
the so-called hazard function ( $H$-function), and the result of the integration of eq.(3) is expressed in the distribution of $\rho$ along $t^{*}$ and the firing rate $\nu$.

It is worth emphasising the rationale behind splitting the input signals into common inputs $(\mathrm{I}(\mathrm{t}), \mathrm{s}(\mathrm{t}))$, which all neurons are subject to and the effects of individual noise for each neuron and in turn how this leads to the $H$-function. We emphasise this because our approach is markedly in contrast to other probability density frameworks, for example, as described in (Apfaltrer et al. (2006)) that leads to a 2D probability density as opposed to ours which results in a 1D probability density. The central idea behind splitting is as follows. First, the common signals can be of any kinetic form. The individual noise can either be instantaneous (e.g. Gaussian white noise) or noise-current (e.g. Ornstein-Uhlenbeck process) and in both cases the noise effect is captured by the hazard function; for derivations see (Chizhov and Graham (2007) and Chizhov and Graham (2008)). Secondly, only voltage fluctuations affect threshold crossing, thus only the fluctuations of membrane potential are considered. The voltage fluctuations around the mean voltage are governed by a linearized stochastic voltage equation, which is the same for different type neurons. The associated Langevin equation is equivalent to its alternative Fokker- Planck (FP) representation (1D for white noise and 2D for colored noise) and is governed by the mean membrane potential $U(t)$ and mean membrane conductance $s(t)$. The hazard function is derived as a solution of the first-time passage problem based on the Fokker-Planck equation for the statistical distribution of voltage fluctuations due to white Gaussian noise near the deterministic mean potential $U$ (Chizhov and Graham (2007)). The problem has exact analytical solutions in two particular cases. In the first case of stationary stimulation, when $U$ and $s$ are constant, the solution is self-similar, with a constant shape distribution for a given value of $U$. Thus the Fokker-Planck equation is reduced to an analytically solvable ordinary differential equation. In the second extreme case of an abrupt excitation with $d U / d t \rightarrow 0$, the solution is obtained from a frozen Gaussian distribution passing the threshold. As shown in (Chizhov and Graham (2007)), a solution for $H$ in an arbitrary case is well approximated by a sum of the two extreme solutions. This solution is universal for different single neuron models; it was approximated with a relatively simple function of $U$ varying in time $t$ at a given $t^{*}$, depending as well on $\sigma_{I}, U_{T}$, and $s(t)$ :

$$
\begin{gathered}
H\left(U\left(t, t^{*}\right), s(t)\right)=\frac{1}{\tau_{m}}\left(A\left(t, t^{*}\right)+B\left(t, t^{*}\right)\right), \\
A\left(t, t^{*}\right)=\exp \left(6.1 \cdot 10^{-3}-1.12 T-0.257 T^{2}-0.072 T^{3}-0.0117 T^{4}\right), \\
B\left(t, t^{*}\right)=-\frac{2 \tau_{m}}{\sqrt{\pi}}\left[\frac{d T}{d t}\right]_{+} \quad \frac{\exp \left(-T^{2}\right)}{1+\operatorname{erf}(T)}, \\
T\left(t, t^{*}\right)=\frac{U_{T}-U\left(t, t^{*}\right)}{\sqrt{2} \sigma_{V}}, \quad \sigma_{V}=\frac{\sigma_{I}}{\sqrt{2 g_{L}\left(g_{L}+s(t)\right)}} .
\end{gathered}
$$

In our simulations, unless otherwise stated, we will consider $U_{T}=0, s(t) \equiv 0$, $\tau_{m}=1$ and $g_{L}=1$. 


\section{Results}

\subsection{Single neuron model: simple bursting neuron}

For the bursting neuron dynamics we consider a modified version of the model developed in Izhikevich (2003) and analysed in Coombes et al. et al. (2012) (using a piecewise-linear approximation). The equations are defined as follows

$$
\begin{aligned}
\frac{d V}{d t} & =|V|-a+I(t)-s(t) V, \\
\tau_{a} \frac{d a}{d t} & =-a, \\
& \text { if } \quad V>V_{\text {th }} \text { then } \quad V=V_{\text {reset }}, \quad a=a+\Delta a,
\end{aligned}
$$

with parameters set to the following values:

$$
V_{\text {th }}=1, V_{\text {reset }}=0.2, \tau_{a}=75, \Delta a=4 / 75, I=0.1 .
$$

These equations define a hybrid model with a planar piecewise-linear (PWL) vector field together with a map, defined by reset conditions for the membrane voltage $V$, as well as an increment for a recovery variable $a$. The fact that $\tau_{a} \gg 1$ induces a timescale separation in the model, where the membrane potential $V$ acts as a fast variable while the recovery variable $a$ is, in comparison, slower. The algebraic conditions (i.e. reset conditions) enable the emergence of limit cycle dynamics in the model, more specifically bursting oscillations (i.e. the dynamics converge to an attracting cycle instead of exploding in finite time). As depicted in Figure 1 (bottom panel), the V-shaped PWL fast nullcline (so-called critical manifold $S_{0}$ of the system) has two branches, the left one is attracting and the right one is repelling. The dynamics along the left (attracting) branch of $S_{0}$ is slow and corresponds to the subthreshold part of the bursting cycle shown in Figure 1 (bottom panel). Outside of the reset zone, the dynamics of the slow variable $a$ is always decaying, which implies that once in the vicinity of the left branch of $S_{0}$ the trajectory moves slowly down towards the corner point of $S_{0}$, where its attractivity changes. Then, as typical in slow-fast systems near points where a one-dimensional critical manifold changes from being attracting to being repelling (where so-called normal hyperbolicity fails), the overall dynamics switch from slow to fast and gets away from $S_{0}$ on the fast timescale.

This transition past, the two reset conditions allow for fast oscillations in between $V_{\text {reset }}$ and $V_{t h}$ as long as the intersection point of the trajectory with the reset line $\left\{V=V_{\text {reset }}\right\}$ stays below the right branch of $S_{0}$, where the flow points rightwards towards the threshold line $\left\{V=V_{t h}\right\}$. However, the reset also affects the slow variable $a$ by incrementing its value each time by a quantity $\Delta a$. After a number of fast oscillations in between $V_{\text {reset }}$ and $V_{t h}$, the intersection point between the trajectory and the reset line crosses the right 
branch of $S_{0}$, where the flow now points leftwards. As a result, the trajectory goes back to the attracting branch of $S_{0}$ where slow dynamics, corresponding to the quiescent phase of the bursting cycle, resumes again.

Note that the notion of threshold for such IF models slightly differs from what is typically meant by threshold in their smooth counterparts, which then corresponds more to the excitability threshold of the membrane model. Indeed, models such as system (6) would explode in finite time without a reset in the membrane potential as it would grow unboundedly past the kink at $V=0$. The threshold value $V_{t h}$ is introduced simply to avoid that the system explodes and to mark the occurrence of a spike (i.e., this is not a strict threshold in neuro-physiological terms). The value $V_{t h}$ is higher than the $V$-level that one would want to associate with the excitability threshold. This is done so as to ensure that multiple spikes can be fired successively, hence mimicking a burst. In the context of smooth bursting models such as the extended MorrisLecar system studied in Terman (1991), the reset value $V_{\text {reset }}$ corresponds to the approximate minimum along the families of limit cycles of the fast subsystem. In such smooth bursters, with a similar geometry as system (6)-(8), the excitability threshold corresponds instead to a small perturbation of the unstable branch of the $V$-nullcline and is well captured by a so-called maximal canard segment (Desroches et al. (2013)). More precisely, for system (6)-(8), the excitability threshold can be approximated by a perturbation of order $1 / \tau_{a}$ of the right branch of the $V$-nullcline.

A complete analysis of the bursting dynamics of system (6)-(8) is beyond the scope of the present work and will be an interesting topic for future work. In particular, it is convenient to focus on slow-fast spike-adding mechanisms present in this model, which allow to understand the burst size and timing. Already, as we will see below, the CBRD method can capture bursting dynamics within a population of neurons of the type given by system (6)-(8) with the noise as in (1). For a population, we set the noise amplitude to be $\sigma_{I}=0.02 \sqrt{2}$ (i.e. $\sigma_{V}=0.02$ ).

\subsection{CBRD model for simple bursting neurons}

To derive the CBRD for bursting neurons we additionally require an extra time parameter, say $t^{* *}$, which denotes the time since last neuronal bursting activity. Relying on the Hybrid model (6)-(8) and its corresponding phaseplane diagram shown in Figure 1, then $t^{* *}$ quantifies the time spent by the trajectory between the termination of the last spike (belonging to the previous burst) until the moment the trajectory reaches the hyperplane $V=0$, corresponding to the apex where the two red nullclines intersect. The time parameter $t^{*}$ has the same meaning as in previous work, where it denotes the time since last spike (i.e. defined as the time moment when $\mathrm{V}$ reaches $V_{t h}$ ). However, we note that during bursting activity, $t^{*}$ can be neglected because realistic neurons are driven towards high-conductance states and as a consequence noise is shunted. The fact that noise is shunted during burst, implies 
that there is no need to evaluate the hazard function and describe the density leak during bursts. Moreover, the neurons become perfectly synchronised within a burst (i.e. the distribution along $t^{*}$ is a delta function). Thus we drop $t^{*}$, but re-name $t^{* *}$ as $t^{*}$ for convenience. To discriminate the neuronal states where the noise is functioning and where it is not, we introduce a discrete state variable $\phi\left(t, t^{*}\right)$ that describes the states, either bursting or quiescent (leak), according to the $(V, a)$-plane (Figure 1$)$. The variable $\phi\left(t, t^{*}\right)$ takes discrete states of either 1 - "leak", obtained when voltage $U\left(t, t^{*}\right)$ decreases below the boundary $U=0$, or 0 - "burst", obtained at the onset of a burst. Moreover, we assume that the slow variable $a$ is reset at $t^{*}=0$ to a some constant or variable $a_{\text {reset }}(t)$ to be given below.

Consequently, the eqs. (3)-(4) for the neuron based on eq. (6)-(7) instead of eq. (1) is obtained as follows:

$$
\begin{aligned}
& \frac{\partial \rho}{\partial t}+\frac{\partial \rho}{\partial t^{*}}=-\rho H(U) \phi \\
& \frac{\partial U}{\partial t}+\frac{\partial U}{\partial t^{*}}=-|U|-a+I(t)-s(t) U+\delta\left(U-V_{t h}\right)\left(V_{\text {reset }}-V_{t h}\right), \\
& \frac{\partial a}{\partial t}+\frac{\partial a}{\partial t^{*}}=-\frac{a}{\tau_{a}}+\delta\left(U-V_{t h}\right) \Delta a, \\
& \frac{\partial \phi}{\partial t}+\frac{\partial \phi}{\partial t^{*}}=-\delta(U) \phi
\end{aligned}
$$

The $H$-function is effective only for $\phi=1$.

The firing rate is defined as a sum of all neurons that reached spike threshold:

$$
\nu(t)=\int_{+0}^{\infty} \rho\left(t, t^{*}\right) \delta\left(U\left(t, t^{*}\right)-V_{t h}\right) d t^{*}
$$

The boundary conditions at the onset of burst are as follows:

$$
\begin{array}{r}
\rho(t, 0)=\int_{+0}^{\infty} \rho H d t^{*}, \\
U(t, 0)=V_{\text {reset }}, \\
a(t, 0)=a_{\text {reset }} \\
\phi(t, 0)=1
\end{array}
$$

The additional parameter is $a_{\text {reset }}$, which approximates $a$ just before a burst if it initiates at the current time $t$. Indeed, $a_{\text {reset }}$ tends to approach the value at the kink point of the red nullclines shown in Figure 1. The kink point is given by the zero of the right hand side of eq. $((6))$ for a single neuron, where $V=0$, as well. From this, we would get $a_{\text {reset }}=I(t)$. However, this condition may lead to fast variation of $a_{\text {reset }}$, if $I(t)$ changes fast. Thus, we introduce an approximation $a_{\text {reset }}=(1+s(t)) U\left(t, t^{*}=\infty\right)$, which is valid 
because $U\left(t, t^{*}=\infty\right)$ is close to the term $I(t) /(1+s(t))$ averaged over time period of 1 (i.e. normalised time period), which follows from the zeroed right hand side of eq.(10).

\subsection{Single neuron model: bursting neuron with potassium current}

As an example of a more complex bursting neuron model with conductancebased description of ionic currents, we add to the model Eqs.(6)-(8) a potassium current $I_{K}(V, t)$, adopted from Yu et al. (2008). For this, eq.(6) is rewritten as

$$
\frac{d V}{d t}=|V|-a+I_{K}(V, t)+I(t)-s(t) V
$$

where the potassium current is approximated as follows

$$
\begin{aligned}
& I_{K}(V, t)=-g_{K} n(V, t)\left(V-V_{K}\right), \\
& \frac{d n}{d t}=\frac{n_{\infty}-n}{\tau_{n}}, \\
& n_{\infty}(V)=\frac{\alpha}{\alpha+\beta}, \quad \tau_{n}(V)=\frac{1}{\alpha+\beta}, \\
& \alpha(V)=\frac{2(-V+0.85)}{\exp ((-V+0.85) / 0.09)-1}, \\
& \beta(V)=\frac{V-0.85}{\exp ((V-0.85) / 0.09)-1} .
\end{aligned}
$$

The integrate-and-fire model requires an additional reset condition:

$$
\text { if } V>V_{t h} \text { then } n=0.5 \text {. }
$$

The parameters are $g_{K}=0.015, V_{K}=-0.3$ and $n_{\text {reset }}=0.5$. After a spike the voltage is partially reset due to the potassium channel, that is why $V_{\text {reset }}$ is set to be larger, $V_{\text {reset }}=0.5$.

\subsection{CBRD model for bursting neurons with potassium current}

Generalization of the CBRD model based on eqs.(9)-(17) to more realistic neurons with explicit approximation of different ionic currents requires to account for the currents in eq.(10) and adding the equations for the ionic channel gating variables. For a population of neurons with potassium channels, the equation 
for the refractory density eq.(9) remains the same and eq.(10) is substituted by

$$
\begin{aligned}
\frac{\partial U}{\partial t}+\frac{\partial U}{\partial t^{*}}= & -|U|-a-g_{K} n\left(V-V_{K}\right)+I(t)-s(t) U \\
& +\delta\left(U-V_{t h}\right)\left(V_{\text {reset }}-V_{t h}\right) .
\end{aligned}
$$

Additionally, the following equation for the potassium gating variable $n$ is derived from eq. $(20)$

$$
\frac{\partial n}{\partial t}+\frac{\partial n}{\partial t^{*}}=\frac{n_{\infty}-n}{\tau_{n}}
$$

with the supplementary expressions eqs.(21), (22) and the boundary condition

$$
n(t, 0)=n_{\text {reset }} \text {. }
$$

The remaining equations are eq.(11)-eq.(17), which completes the description. Note that the parameterization of $n$ by $t^{*}$ helped to preserve the dimension of PDEs.

\subsection{Simulations}

Simulations with the CBRD-model (eqs. (3)-(5)) for the LIF neuron population are shown in (Fig. 2). Here we test the CBRD by considering two test problems, namely, stimulation with a current step and a complex input, which demonstrate that $\mathrm{CBRD}$ accurately reproduces the solutions obtained with the Monte-Carlo simulations (Fig. 2). As an example of a complex-shaped input, we consider a sinus with an increasing frequency (Fig. 2D) as the current $I(t)$, and the absolute function of similar sinus-function with twice smaller frequency as the conductance $s(t)$ (Fig. 2D). Note that the model performs well in the case of stimulation by both the voltage independent current $I(t)$ and the conductance $s(t)$.

We subsequently simulate the CBRD-model for simple bursting neurons (eqs. (9)-(17)) for a test problem of a current step stimulation (Fig. 3A). The firing rate as a population response to the stimulus is shown in Figure 3C. The corresponding voltage response of an individual neuron is depicted in Figure 3B. Due to synchronized initial state of the neurons within the population the first burst turns out to be hyper-synchronous. Peaks of the firing rate during the first burst correspond to separate spikes. At the interval between the first and second burst, neuronal states desynchronize, which gives rise to a smoothing effect of the firing rate in subsequent bursts. In fact, this smoothing effect amplifies in subsequent bursts and thus a total desynchronisation occurs. The firing rate obtained with the population model is compared to the one from Monte-Carlo simulation (Fig. 3C), which shows satisfactory agreement, though showing weaker fading of the response oscillations. An important property of the proposed model is that the timing of the population firing rate bumps are reproduced accurately. 
We note that the voltage $U$ does not depend on $\nu$. As a consequence, for constant step stimulation, the voltage profile in $t^{*}$-space is stationary (Fig. 4 B). The corresponding time-profile in $t^{*}$-space is shown (as green) in Fig. 4 C. At $t^{*}$ close to $100 \mathrm{~ms}$, the voltage approaches the threshold (orange). As a consequence, and according to eq. (5), the hazard function increases (blue). As expected, this implies that a significant fraction of neurons with $t^{*}$ close to $100 \mathrm{~ms}$ switch to the bursting phase, upon which there is a reset of $t^{*}$ (i.e. $\left.t^{*}=0\right)$. Moreover, according to eq. (9), the neuronal density (red) decreases with $t^{*}$ at $t^{*}$ close to $100 \mathrm{~ms}$ and increases at $t^{*}=0$. At $t^{*}$ from 0 until about $80 \mathrm{~ms}$, the neuronal density shifts to the right and then decays. Note that because the flux to the reset point $t^{*}=0$ affects $\rho$ and depends on it, the neuronal density converges to the steady state much slower than the voltage, as seen from the comparison between Fig. $4 \mathrm{~A}$ and Fig. 4 B.

The proposed model works with an arbitrary input. Again, we consider a complex-shaped input with variable current $I(t)$ and conductance $s(t)$ (Fig. 3D), as in Fig. 2D. A single neuron fires irregularly in response to such input (Fig. 3E). The firing rate obtained with the population model is well compared to the one from Monte-Carlo simulation (Fig. 3F) in respect to qualitative behaviour with fading oscillations, the amplitudes of the firing rate peaks and the timing of the peaks. The residual difference is due to the approximate nature of two assumptions: the one about $a_{\text {reset }}$ and the other one that neglects by the effects of noise during the bursts. Note that the second assumption would be more relevant to more realistic neurons with shunting, for which the noise effect vanishes at the high conductance state during bursts.

Finally, we demonstrate that the CBRD model for bursting neurons with potassium current have similar qualitative behaviour (Fig. 5). The effect of the potassium current on voltage is seen from comparison of the traces from the insets in Figs. 3B and 5B. After spikes, the voltage is partially reset due to the potassium channel. The potassium current is reset at each spike and it vanishes between the spike bursts. Qualitatively, the bursts are similar to those in the simple bursting model. The firing rate is similar to that for the simple bursting neuron population.

All together, these derivations and simulations demonstrate that CBRD framework can be extended to a population of intrinsically bursting neurons.

\subsection{Discussion}

This is a preliminary computational study that demonstrates the plausibility of extending CBRD framework towards bursting dynamics. There are however a number of mathematical challenges (and further computational ones), as well as experimental validations to consider in the near future. From the mathematical viewpoint, it will be essential to analyse the properties of the nonlinear renewal system of equations (9)-(11), in particular its asymptotic time behavior (Perthame and Tumuluri , 2008; Cañizo and Yoldaş , 2018). 
Additionally, it will be interesting to bridge the CBRD (i.e. PDEs) proposed in the present paper with detailed piecewise deterministic Markov processes (PDMP) approaches where spike trains events are explicitly described with the help of stochastic point processes (Chevallier et al. , 2015). Indeed, system (9)(11) is the large population mean-field approximation of systems of coupled PDMP models. This will help to assess the stochastic disparity between the two approaches in terms of martingale problems. In this perspective, an intermediate model could be proposed, as a stochastic PDE, using a diffusion approximation (Dumont et al. , 2017).

Another future direction for the present work will be to enhance the slowfast analysis of the single neuron model used in the population density model that we have proposed here. Indeed, CBRD for bursting models allows to finely analyse both the quiescent and the burst phases. In particular, their duration and timing can be more precisely estimated by combining the knowledge of the fast subsystem's bifurcation structure together with the slow oscillation that passes through it in the full system. Besides, this analysis can be simplified when the underlying model is piecewise-linear (Desroches et al. (2016)). Such a slow-fast study will shed further light onto the role of the two main parameters of the density model, namely time since last spike and time since last burst. Finally, the interactions between slow-fast timescales and stochasticity can be analysed using a mix of CBRD and probability theory (Berglund and Gentz (2006)); we plan to extend the theoretical aspects of the modelling framework proposed in the present work by using such methods.

From a biophysics point of view, it will be worth to re-interpret the macroscopic neuronal density. In particular, note that the starting point of the CBRD is the assumption of a statistical ensemble of uncoupled neurons (possibly differentiated by noise). This assumption of uncoupled neurons, invokes the Boltzmann molecular chaos hypothesis (also called Stosszahlansatz), where the collision of particles (effectively the coupling) is neglected. This is justified by the fact that the collisions are elastic and, due to conservation laws, the colliding gas particles effectively forget the effects of the previous collisions (i.e. collisions de-correlate exponentially fast). In this view, there is the tantalising question as to what would be the corresponding conservation law (if any) and in what physiological conditions would it be valid.

The proposed model has been validated by comparison with an alternative model, the Monte-Carlo simulations. From an experimental point of view, it will be also important to validate the CBRD for bursting dynamics. We recall that the CBRD for spiking dynamics has been validated against data. Specifically, in a previous work carried out in Chizhov (2017), a CBRD model for adaptive, regular spiking neurons was compared with electrophysiological recordings in a single pyramidal cell, obtained by Tchumatchenko et al. (Tchumatchenko et al. (2011)). These experiments were in whole-cell patch-clamp recordings and the perturbation protocol followed a time-varying weak piecewise current (i.e. changing step-wise current) that was injected into the neuron. The statistics of spiking response were characterized by the post-stimulus spike-time histogram, which in the CBRD framework corresponds to the fir- 
ing rate for a neuronal population. In simulations, the spike trains in response to stepwise current injection revealed that the CBRD model responses replicate and explain experimental neuronal responses, including the effects of the adaptation. Taking these experiments as a starting point, we propose similar future experiments for bursting cells, which we believe is feasible but has not yet been done. These envisaged experiments could then be compared to the bursting CBRD approach.

The probability density approach (PDA), and particularly the refractory density approach are an important class of modelling framework that have potential to solve neurobiological questions. For instance, a PDA-based model of a single population revealed the effects of synaptic noise filtering by LIF neurons (Brunel et al. , 2001). A problem of gain modulation in refractory integrate-and-fire neurons receiving an input with shunt-dependent fluctuations has been solved with the help of a PDA-based model (Ly and Doiron , 2009). Networks of coupled excitatory and inhibitory integrate-and-fire neurons have been simulated with a PDA (Nykamp and Tranchina, 2000; Apfaltrer et al. , 2006). The CBRD approach refines the PDA approach and has further shown to be well suited to efficiently study cortical activity and also to have good agreements with some experiments. For example, the CBRD has been applied to study the visual cortex activity (Chizhov , 2014), where a cortical domain was considered as a layered continuum of interacting excitatory and inhibitory neuronal populations. The simulations allowed to reproduce evoked responses of the cortical network that were registered by the patchclamp electrophysiological methods and the optical recordings performed in vivo. Another study with a CBRD model has revealed mechanisms of epileptic interictal discharges (Chizhov et al. , 2017). In this study, the quality of the CBRD method was important to reproduce abrupt onset of spontaneous pulses of hyper-synchronized activity as observed by electrophysiological measurements. Aspects of spatial propagation of the interictal discharges have been further simulated in Chizhov et al. (2019) and found to be consistent with paired pach-clamp recordings.

The CBRD approach proposed for simplified bursting neurons challenges a future derivation of more sophisticated population activity models for HodgkinHuxley-like bursting neurons, which will enable modeling of synchronised bursting activity in hyper-excitable brain states as epilepsy. It is well-known that an increase in intrinsic excitability can cause bursting in cells which usually fire single action potentials. Extracellular potassium concentration has been shown to modulate intrinsic excitability (Jensen and Yaari (1997)). It is well established that the extracellular potassium concentration increases during epileptogenesis and may be critically involved in synchronized burst oscillations during seizures. Moreover, nonsynaptic, spontaneous activity switches from single spikes to bursting when the concentration is increased (Frohlich and Bazhenov (2006)). With the proposed model, an application of the CBRD approach to study epilepsy may be extended to the case of significant contribution of intrinsically bursting cells. 


\section{References}

Amari SI (1977) Dynamics of pattern formation in lateral-inhibition type neural fields. Biol Cybern 27(2):77-87

Apfaltrer F, Ly C, Tranchina D (2006) Population density methods for stochastic neurons with realistic synaptic kinetics: firing rate dynamics and fast computational methods. Network 17(4):373-418

Avitabile D, Desroches M, Knobloch E (2016) Spatiotemporal canards in neural field equations. Phys Rev E 95(4): 042205

Berglund N, Gentz B (2006) Noise-induced phenomena in slow-fast dynamical systems: a sample-paths approach. Springer

Breakspear M, Roberts J. A., Terry J. R., Rodrigues S, Mahant N, Robinson P. A. (2015) A unifying explanation of primary generalized seizures through nonlinear brain modeling and bifurcation analysis. Cerebral Cortex 16(9), 1296-1313

Brunel N, Hakim H (1999) Fast global oscillations in networks of integrate-andfire neurons with low firing rates. Neural Computation 11(7), 1621-1671

Brunel N, Chance FS, Fourcaud N, Abbott LF (2001) Effects of synaptic noise and filtering on the frequency response of spiking neurons. Phys Rev Lett. 86(10):2186-2189

Cañizo JA, Yoldaş H (2018) Asymptotic behaviour of neuron population models structured by elapsed-time. ArXiv e-prints arXiv:1803.07062

Casti ARR, Omurtag A, Sornborger A, Kaplan E, Knight B, Victor J, Sirovich L (2002) A population study of integrate-and-fire-or-burst neurons. Neural Computation 14: 957-986

Chevallier J, Cáceres MJ, Doumic M, Reynaud-Bouret P (2015) Microscopic approach of a time elapsed neural model. Math Models Methods Appl Sci 25(14): 2669-2719

Chizhov AV (2014) Conductance-based refractory density model of primary visual cortex. J Comput Neurosci 36(2):297-319

Chizhov AV (2017) Conductance-Based Refractory Density Approach: Comparison with Experimental Data and Generalization to Lognormal Distribution of Input Current. Biol Cybernetics 111(5-6):353-364

Chizhov AV, Amakhin DV, Zaitsev AV (2017) Computational model of interictal discharges triggered by interneurons. PLoS One 12(10):e0185752

Chizhov AV, Amakhin DV, Zaitsev AV (2019) Spatial propagation of interictal discharges along the cortex. Biochemical and Biophysical Research Communications 508: 1245-1251

Chizhov AV, Amakhin DV, Zaitsev AV (2019) Mathematical model of Na-K$\mathrm{Cl}$ homeostasis in ictal and interictal discharges. Plos One 14(3): e0213904. 
Chizhov AV, Graham LJ (2007) Population model of hippocampal pyramidal neurons, linking a refractory density approach to conductance-based neurons. Phys Rev E 75:011924

Chizhov AV, Graham LJ (2008) Efficient evaluation of neuron populations receiving colored-noise current based on a refractory density method. Phys Rev E 77:011910

Chizhov AV, Graham LJ, Turbin AA (2006) Simulation of neural population dynamics with a refractory density approach and a conductance-based threshold neuron model. Neurocomputing 70:252-262.

Chizhov AV, Rodrigues S, Terry JR (2007) A comparative analysis of a detailed neural population model and a mean-field EEG model. Phys Lett A 369:31-36

Coombes S, Thül R, Wedgwood KCA (2012) Nonsmooth dynamics in spiking neuron models. Physics D 241:2042-2057.

Desroches M, Guillamon A, Ponce E, Prohens E, Rodrigues S, Teruel AE (2016) Canards, folded nodes, and mixed-mode oscillations in piecewiselinear slow-fast systems. SIAM Rev 58(4):653-691

Desroches M, Kaper TJ, Krupa M (2013) Mixed-mode bursting oscillations: Dynamics created by a slow passage through spike-adding canard explosion in a square-wave burster. Chaos 23(4):046106

Desroches M, Rodrigues S (2018) Classification of bursting patterns: Review \& Extensions. submitted preprint

Dumont G, Payeur A, Longtin A (2017) A stochastic-field description of finitesize spiking neural networks. PLOS Comput Biol 13(8):e1005691

Eggert J, van Hemmen JL (2001) Modeling neuronal assemblies: Theory and implementation. Neural Computation 13:1923-1974

Fenichel N (1979) Geometric singular perturbation theory for ordinary differential equations. J Differential Equation 31(1):53-98

Ferguson K. A., Njap F., Nicola W., Skinner F. K., Campbell S. A (2015) Examining the limits of cellular adaptation bursting mechanisms in biologically-based excitatory networks of the hippocampus. Journal of Computational Neuroscience 39(3):289-309

Fernandez FR, White JA (2010) Gain control in CA1 pyramidal cells using changes in somatic conductance. J Neuroscience 30(1):230-241

Fourcaud N, Brunel N (2002) Dynamics of the firing probability of noisy integrate-and-fire neurons, Neural Computation 14(9):2057-110

Freeman W (1972) Waves, pulses, and the theory of neural masses. Progress in Theoretical Biology 2(1)

Freeman W (1975) Mass action in the nervous system. Academic Press

Harrison LM, David O, Friston KJ (2005) Stochastic models of neuronal dynamics. Philos Trans R Soc Lond B Biol Sci. 360(1457):1075-91

Fröhlich F, Bazhenov M (2006) Coexistence of tonic firing and bursting in cortical neurons. Phys Rev E 74:031922 
Gerstner W, Kistler WM (2002) Noise in spiking neuron models, in Spiking Neuron Models: Single Neurons, Populations, Plasticity. Cambridge University Press, pp.147-200

Gerstner W, Kistler WM, Naud R, Paninski L (2014) Introduction: neurons and mathematics, in Neuronal Dynamics. From Single Neurons to Networks and Models of Cognition. Cambridge University Press, pp.395-416

Gerstner W, Naud R (2009) How good are neuron models? Science 326, $379-326$

beim Graben P, Rodrigues S (2013) A biophysical observation model for field potentials of networks of leaky integrate-and-fire neurons. Front Comput Neurosci 6:100

Hansel D, Sompolinsky H (1998) Modeling feature selectivity in local cortical circuits. In C. Koch and I. Segev (Eds.), Methods in neuronal modeling: From synapses to networks, 2nd Edition. Cambridge, MA: MIT Press, pp. 499-567

Izhikevich EM (2000) Neuronal excitability, spiking and bursting. Int J Bifur and Chaos 10(6):1171-1266

Izhikevich EM (2003) Simple model of spiking neurons. IEEE Trans Neural Networks 14(6):1569-1572

Jensen MS, Yaari Y (1997) Role of intrinsic burst firing, potassium accumulation, and electrical coupling in the elevated potassium model of hippocampal epilepsy. J Neurophysiol 77(3):1224-1233

Johannesma PIM (1968) Diffusion models of the stochastic activity of neurons. In Neural Networks. Springer, Berlin, pp.116-144.

Jones CKRT (1995) Geometric singular perturbation theory. In: Arnold L, Jones CKRT, Mischaikow K, Raugel G (eds) Dynamical systems, Lect Notes in Math 1609:44-118, Springer, Berlin, Heidelberg

Knight B (1972) Dynamics of Encoding in a Population of Neurons. The Journal Of General Physiology 59:734-766

Knight BW, Manin D, Sirovich L (1996) Dynamical models of interacting neuron populations. In: Gerf E C (ed) Symposium on Robotics and Cybernetics: Computational Engineering in Systems Applications. Cite Scientifique, Lille, France

Knight BW, Omurtag A, Sirovich L (2000) The Approach of a Neuron Population Firing Rate to a New Equilibrium: An Exact Theoretical Result. Neural Computation 12:1045-1055

Ly C, Doiron B (2009) Divisive Gain Modulation with Dynamic Stimuli in Integrate-and-Fire Neurons. PLoS Comput Biol 5(4): e1000365

Ly C, Tranchina D (2007) Critical analysis of dimension reduction by a moment closure method in a population density approach to neural network modeling. Neural computation 19(8): 2032-2092 
Ly C, Tranchina D (2009) Spike train statistics and dynamics with synaptic input from any renewal process: a population density approach. Neural computation 21: 360-396

Marten F, Rodrigues S, Benjamin O, Richardson MP, Terry JR (2009) Onset of polyspike complexes in a mean-field model of human electroencephalography and its application to absence epilepsy. Philosophical Transactions of the Royal Society A: 367(1891):1145-1161

Montbrió E, Pazó D, Roxin A (2015) Macroscopic Description for Networks of Spiking Neurons. Phys. Rev. X 5: 021028

Moreno-Bote R, Parga N (2005) Membrane potential and response properties of populations of cortical neurons in the high conductance state. Phys Rev Lett 94(8):088103

Nykamp DQ, Tranchina D (2000) A population density approach that facilitates large-scale modeling of neural networks: Analysis and an application to orientation tuning. J Comput Neurosci 8:19-50

Ott E, Antonsen TM (2008) Low dimensional behavior of large systems of globally coupled oscillators. Chaos 18(3):037113

Perthame B, Tumuluri SK (2008) Nonlinear renewal equations. In: Bellomo N, de Angelis E (eds), Selected Topics in Cancer Modeling, Springer

Rinzel J (1987) A formal classification of bursting mechanisms in excitable systems. In International Congress of Mathematicians, Berkeley, California, USA, August 3-11, 1986, volume II, pp. 1578-1593. American Mathematical Society

Rodrigues S., Barton D., Szalai R., Benjamin O., Richardson M. P., Terry J. R. (2009) Transitions to spike-wave oscillations and epileptic dynamics in a human cortico-thalamic mean-field model Journal of Computational Neuroscience 27(3):507-526

Roxin A, Brunel N, Hansel D (2005) Role of delays in shaping spatiotemporal dynamics of neuronal activity in large networks. Phys Rev Lett 94(23):238103

Schwalger T, Deger M, Gerstner W (2017) Towards a theory of cortical columns: From spiking neurons to interacting neural populations of finite size. PLoS Comput Biol 13(4):e1005507

Smirnova EY, Zaitsev AV, Kim KKh, Chizhov AV (2015) The domain of neuronal firing on a plane of input current and conductance. J Comput Neurosci 39(2):217-33

Tchumatchenko T, Malyshev A, Wolf F, Volgushev M (2011) Ultrafast population encoding by cortical neurons. J Neurosci 31(34):12171-12179

Terman D (1991) Chaotic spikes arising from a model of bursting in excitable membranes. SIAM J Appl Math 51(5):1418-1450

Nykamp D, Tranchina D (2000) A population density approach that facilitates large-scale modeling of neural networks: Analysis and application to 

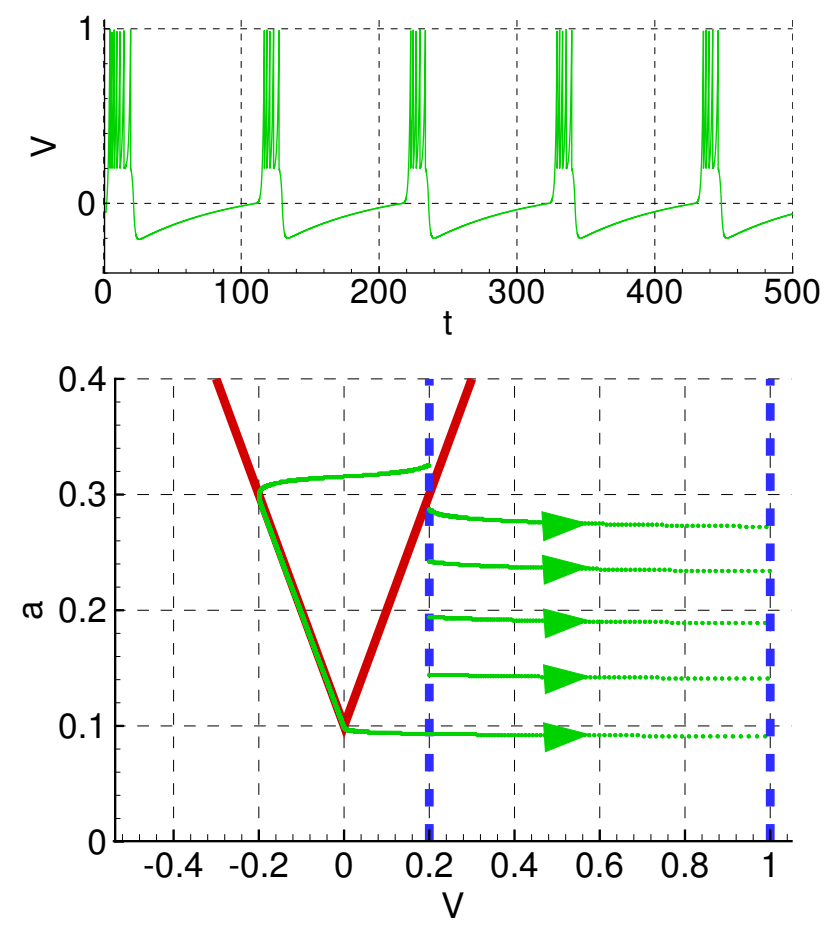

Fig. 1 Hybrid model for bursting neuron. Voltage trace (top) and phase-space plot (bottom) with nullclines (red and blue) and a trajectory (green). The red nullcllines intersect at hyperplane $V=0$, which corresponds to the true neurophysiological threshold (seen from the perspective of smooth continuous time slow-fast systems). The dashed blue line (left) correspond to the $V_{\text {reset }}$ and the dashed blue line (right) correspond to $V_{t h}$.

orientation tuning. J Comput Neuroscience 8:19-50

Ventriglia F (1974) Kinetic approach to neural systems: I. Bull Math Biol $36(5-6): 535-544$

Wilson HR, Cowan JD (1972) Excitatory and inhibitory interactions in localized populations of model neurons. Biophys J 12(1):1-24

Yu Y, Shu Y, McCormick DA (2008) Cortical action potential back propagation explains spike threshold variability and rapid-onset kinetics. J Neuroscience 28(29):7260-7272 

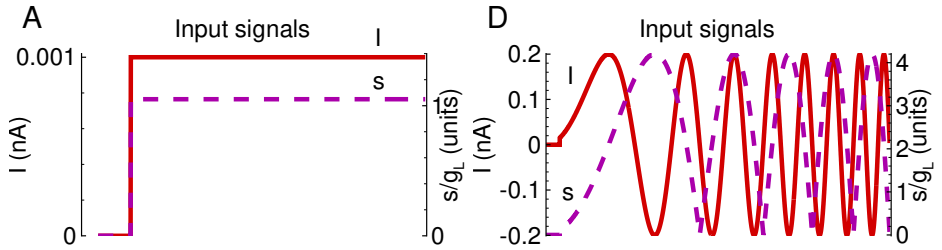

$\mathrm{B}$

$\mathrm{E}$
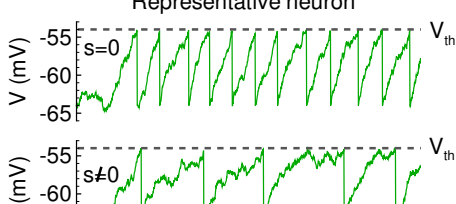

$\mathrm{V}_{\mathrm{th}}$

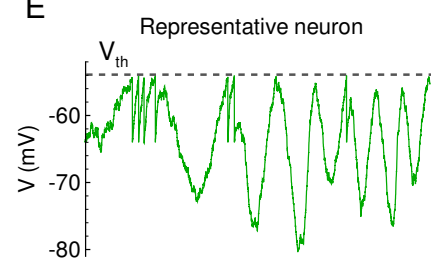

\section{C}

Population Firing Rate

$\mathrm{F}$
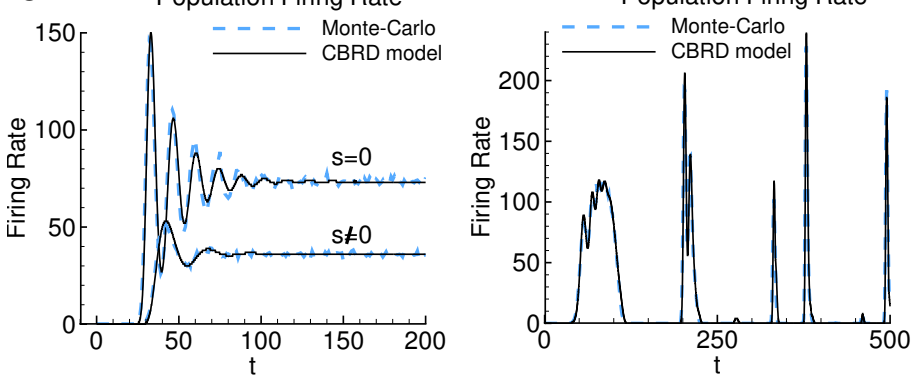

Fig. 2 Population of LIF neurons. A-C: Response to step-wise stimuli. $D$-F: Response to a complex-shaped current. $A, D$ : Input current $I(t)$ and conductance $s(t)$. $B, E$ : Membrane voltage of a single neuron. $C, F$ : Population firing rate, calculated in Monte-Carlo simulation and the CBRD model. 50000 trials were used in the Monte-Carlo simulations. 

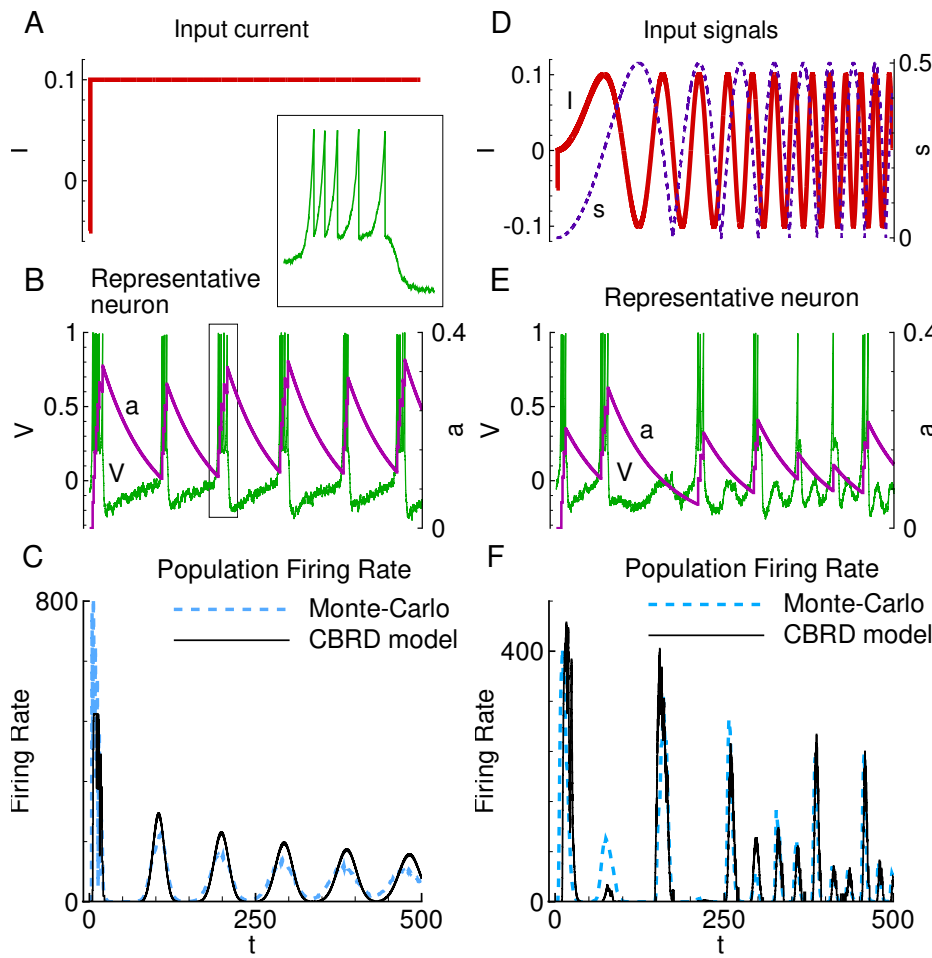

E Representative neuron
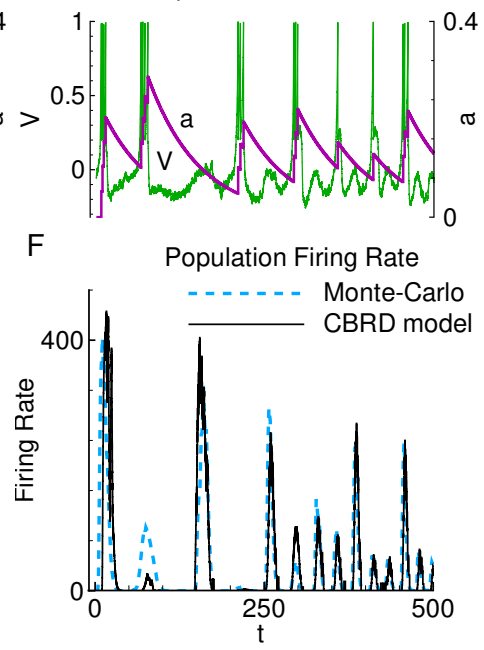

Fig. 3 Population of bursting neurons. A-C:Response to a step of current. $D-F$ : Response to a complex-shaped current. $A, D$ : Input current $I(t)$ and conductance $s(t)$. B,E: Membrane voltage of a single neuron. The green traces are the voltage responses, while the time trajectory of the recovery variable, $a$ is shown in purple. $C, F$ : Population firing rate, calculated in Monte-Carlo simulation and the CBRD model. 20000 trials were used in the Monte-Carlo simulations. 
$A$

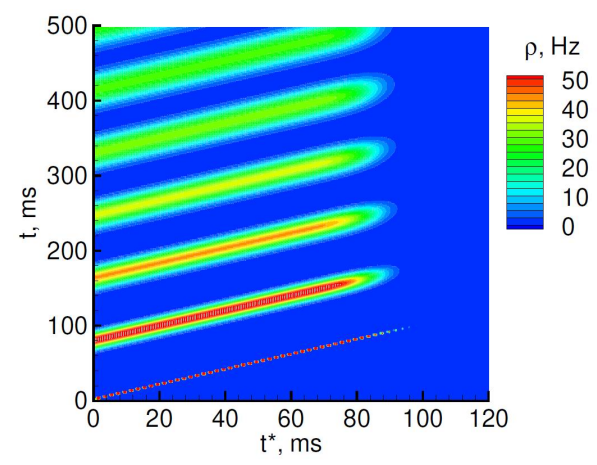

$B$

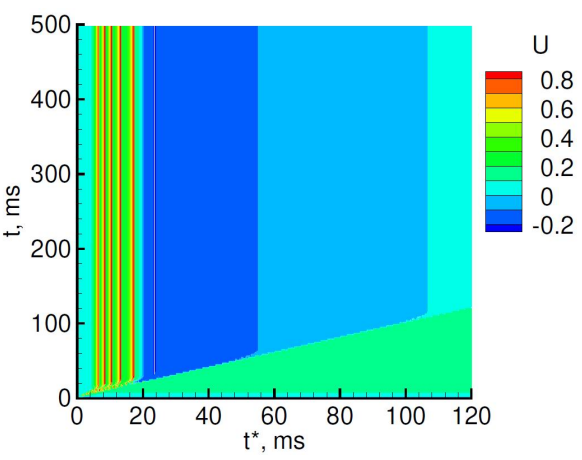

$C$
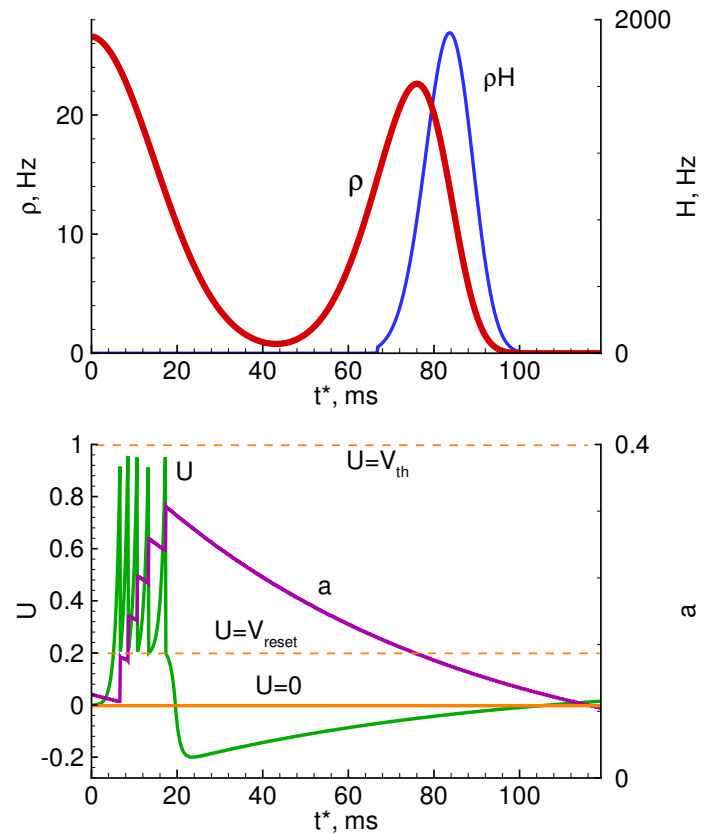

Fig. 4 This plot extends Fig. 3A-C. Specifically, it depicts $t^{*}-t$-plots for the neuronal density $\rho(A)$ and voltage $U(B)$, that lead to the firing rate response shown in Fig. 3C. Panel $C$ shows the profiles in $t^{*}$-space at $t=500 \mathrm{~ms}$ for the neuronal density $\rho$ and the source term $\rho H$ (middle panel), and the mean voltage $U$ and adaptation $a$ (bottom panel). 

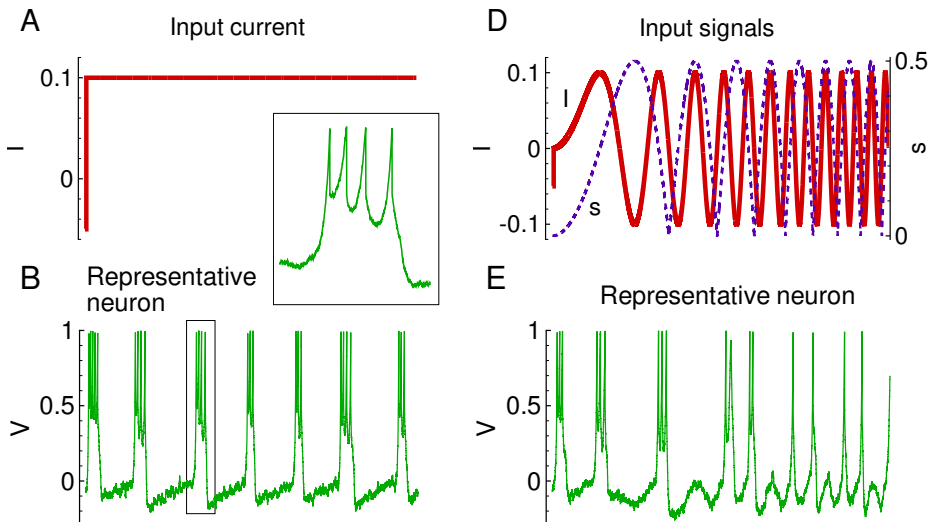

E Representative neuron
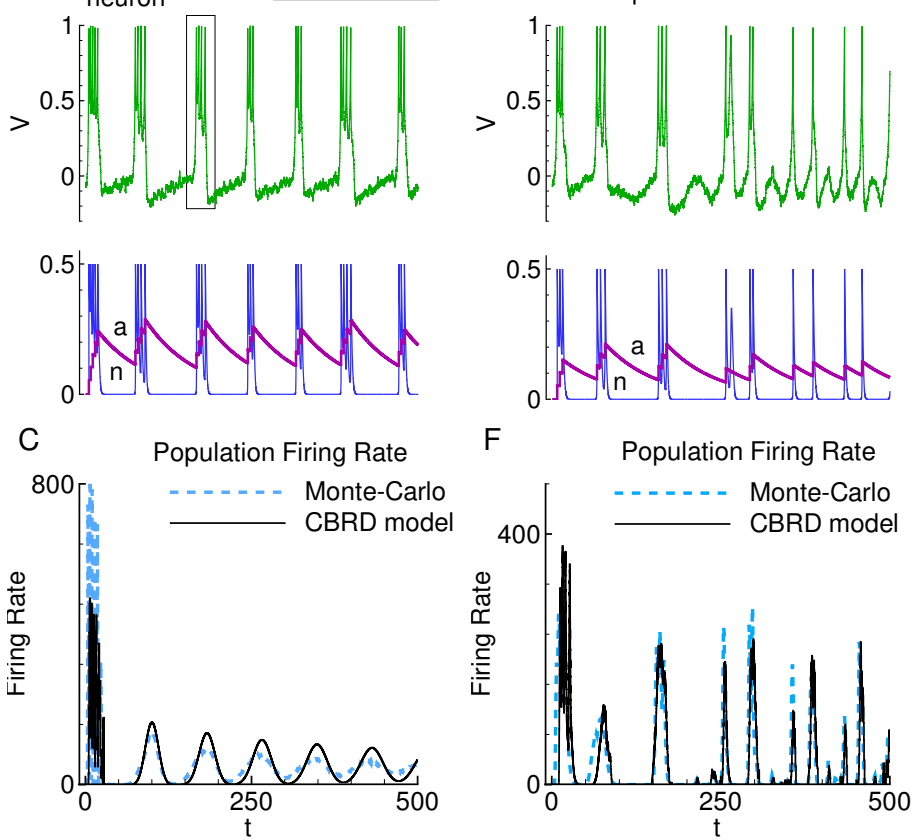

Fig. 5 Population of bursting neurons with potassium current. A-C:Response to a step of current. $D-F$ : Response to a complex-shaped current. $A, D$ : Input current $I(t)$ and conductance $s(t)$. B,E: Membrane voltage of a single neuron. The green traces are the voltage responses, while the time trajectory of the recovery variable, $a$ and the potassium gating variable $n$ are shown in purple and blue, correspondingly. C,F: Population firing rate, calculated in Monte-Carlo simulation and the CBRD model. 20000 trials were used in the Monte-Carlo simulations. 